appeared in:

Hyperbolic problems: theory, numerics and applications, pp. 337-346. Proceedings of Symposia in Applied Mathematics, Volume 67, Part 2. American Mathematical

Society, Providence 2009.

\title{
Two-dimensional transport equation with Hamiltonian vector fields
}

\author{
Giovanni Alberti, Stefano Bianchini, and Gianluca Crippa
}

\begin{abstract}
We illustrate the main steps in the proof of a sharp result of well-posedness for the two-dimensional transport equation whose vector field is bounded, autonomous, divergence free and satisfies the so-called "weak Sard property". We also remark on the fact that the weak Sard property we identify is indeed equivalent to the well-posedness.

Keywords: Transport equation, two-dimensional transport equation, Sard lemma, Hamiltonian function, coarea formula

Mathematics Subject Classification (2000): 35F10 (35A05, 28A80)
\end{abstract}

\section{Introduction and main notation}

The study of the well-posedness of the transport equation

$$
\left\{\begin{array}{l}
\partial_{t} u(t, x)+b(t, x) \cdot \nabla u(t, x)=0 \\
u(0, x)=\bar{u}(x)
\end{array}\right.
$$

where $b:[0, T] \times \mathbb{R}^{d} \rightarrow \mathbb{R}^{d}$ is a vector field, $\bar{u} \in L^{\infty}\left(\mathbb{R}^{d}\right)$ is the initial data, and the unknown $u$ belongs to $L^{\infty}\left([0, T] \times \mathbb{R}^{d}\right)$, is of great importance in the theory of nonlinear evolutionary partial differential equations, due to the appearance of equations of this form in many physical phenomena; we refer for instance to [16] and [26] for a general overview on the theory of conservation laws. The theory is classical and well-understood in the case when $b$ is sufficiently smooth (at least Lipschitz with respect to the spatial variable, uniformly with respect to the time), and is strongly based on the so-called theory of characteristics, i.e. on the connection between (1.1) and the ordinary differential equation

$$
\left\{\begin{array}{l}
\dot{\gamma}(t)=b(t, \gamma(t)) \\
\gamma(0)=x
\end{array}\right.
$$

However, in many applications motived by physical models, non-smooth vector fields show up as velocity fields of transport equations. Thus a great interest has arisen in the study of (1.1) when $b$ is only in some classes of weak differentiability. We mention in this context the two seminal papers by DiPerna and Lions [20] and by Ambrosio [3], in which the Sobolev and the $B V$ cases respectively are considered, in both cases under boundedness assumptions on the spatial divergence of the vector field. The result in the $B V$ framework has been applied in [6] and [5], obtaining well-posedness results for the Keyfitz-Kranzer system [25]. Various counterexamples (see for instance [19], and also [9] for a related example in the context of the two-dimensional Keyfitz-Kranzer system) show that some weak differentiability assumptions on the vector field are in general necessary in order to obtain well-posedness. For a general survey on this topic see for instance [7]. 
In this note we describe some recent results obtain by the authors in [2], regarding the two-dimensional case. Let us consider for simplicity the basic case of an autonomous two-dimensional divergence free vector field with compact support:

$$
b \in L^{\infty}\left(\mathbb{R}^{2} ; \mathbb{R}^{2}\right), \quad \operatorname{div} b=0 \quad \text { and } \quad \operatorname{spt} b \subset \subset \mathbb{R}^{2} .
$$

It is well-known that in this situation it is possible to find a Hamiltonian function $H \in \operatorname{Lip}_{c}\left(\mathbb{R}^{2}\right)$ such that

$$
b(x)=\nabla^{\perp} H(x)=\left(-\frac{\partial H(x)}{\partial x_{2}}, \frac{\partial H(x)}{\partial x_{1}}\right) \quad \text { for } \mathscr{L}^{2} \text {-a.e. } x \in \mathbb{R}^{2} .
$$

Due to this very particular structure, we intuitively expect that the assumptions needed for the well-posedness should be dramatically weaker than the previous ones. The starting point for this hope is the heuristic remark that the value of the Hamiltonian is constant on the trajectories. Indeed, if $\dot{\gamma}(t)=b(\gamma(t))$, then we can compute

$$
\frac{d}{d t} H(\gamma(t))=\nabla H(\gamma(t)) \cdot \dot{\gamma}(t)=\nabla H(\gamma(t)) \cdot b(\gamma(t))=\nabla H(\gamma(t)) \cdot \nabla^{\perp} H(\gamma(t))=0 .
$$

This means that the trajectories "follow" the level sets of the Hamiltonian. Going on with heuristics, one would try to implement the following strategy:

(a) Localize the equation to each level set, thanks to the fact that the level sets are invariant under the action of the flow;

(b) Understand the structure of the level sets, trying to prove that generically they are "one-dimensional sets";

(c) See the equation on each level set as a one-dimensional problem and show uniqueness for it;

(d) Deduce uniqueness for the problem in $\mathbb{R}^{2}$ from the uniqueness of all the problems on the level sets.

Since we can hope for uniqueness on the level sets under quite general hypotheses, the reduced equation being one-dimensional, we expect stronger well-posedness results in this case: it is natural to imagine that no regularity of $b$ (in terms of weak derivatives) would be needed.

We shall see in the following (see in particular Theorem 6.2) that in this context the well-posedness is ensured by a very weak condition regarding the critical points of the Hamiltonian $H$, namely by the weak Sard property (4.1). In fact, we are also able to show that (4.1) is necessary and sufficient for the well-posedness.

We close this first section by indicating the main notation used in the sequel. We denote by $\mathscr{B}\left(\mathbb{R}^{d}\right)$ the family of the Borel subsets of $\mathbb{R}^{d}$, by $\mathscr{M}\left(\mathbb{R}^{d}\right)$ the family of the locally finite Borel measures on $\mathbb{R}^{d}$ and by $\mathscr{M}_{+}\left(\mathbb{R}^{d}\right)$ the subset of $\mathscr{M}\left(\mathbb{R}^{d}\right)$ consisting of all nonnegative locally finite Borel measures on $\mathbb{R}^{d}$.

If $\mu \in \mathscr{M}\left(\mathbb{R}^{d}\right)$ and $E \subset \mathbb{R}^{d}$ is a Borel set, the restriction of $\mu$ to $E$ is the measure $\mu\left\llcorner E \in \mathscr{M}\left(\mathbb{R}^{d}\right)\right.$ defined by $(\mu\llcorner E)(A)=\mu(A \cap E)$ for every Borel set $A \subset \mathbb{R}^{d}$. If $\mu \in \mathscr{M}\left(\mathbb{R}^{d}\right)$ and $\nu \in \mathscr{M}_{+}\left(\mathbb{R}^{d}\right)$ we say that $\mu$ is absolutely continuous with respect to $\nu$ (and we write $\mu \ll \nu$ ) if $|\mu|(E)=0$ for every Borel set $E \subset X$ such that $\nu(E)=0$. We say that two measures $\mu$ and $\nu \in \mathscr{M}\left(\mathbb{R}^{d}\right)$ are mutually singular (and we write $\mu \perp \nu$ ) if they are concentrated on disjoint Borel sets. For $\mu \in \mathscr{M}\left(\mathbb{R}^{d}\right)$ and $\nu \in \mathscr{M}_{+}\left(\mathbb{R}^{d}\right)$ we recall that the Lebesgue decomposition theorem gives the existence of $\mu^{a} \in \mathscr{M}\left(\mathbb{R}^{d}\right)$ and $\mu^{s} \in \mathscr{M}\left(\mathbb{R}^{d}\right)$ such that

$$
\mu^{a} \ll \nu, \quad \mu^{s} \perp \nu \quad \text { and } \quad \mu=\mu^{a}+\mu^{s} .
$$


If $f: \mathbb{R}^{d} \rightarrow \mathbb{R}^{m}$ is a Borel map and $\mu \in \mathscr{M}\left(\mathbb{R}^{d}\right)$ we denote by $f_{\#} \mu \in \mathscr{M}\left(\mathbb{R}^{m}\right)$ the push-forward of the measure $\mu$, defined by

$$
\left(f_{\#} \mu\right)(E)=\mu\left(f^{-1}(E)\right) \quad \text { for every Borel set } E \subset \mathbb{R}^{m} .
$$

We denote by $\mathscr{L}^{d}$ the Lebesgue measure on $\mathbb{R}^{d}$ and with $\mathscr{H}^{k}$ the $k$-dimensional Hausdorff measure on $\mathbb{R}^{d}$. We say that a Borel set $\Sigma \subset \mathbb{R}^{d}$ is $\mathscr{H}^{k}$-rectifiable if there exist countably many Lipschitz functions $f_{i}: \mathbb{R}^{k} \rightarrow \mathbb{R}^{d}$ such that

$$
\mathscr{H}^{k}\left(\Sigma \backslash \cup_{i} f_{i}\left(\mathbb{R}^{k}\right)\right)=0 .
$$

We finally recall the coarea formula for Lipschitz functions, which shall be frequently used in our analysis: for every Lipschitz map $f: \mathbb{R}^{d} \rightarrow \mathbb{R}^{d-k}$ and every positive Borel function $\varphi: \mathbb{R}^{d} \rightarrow[0,+\infty]$ there holds

$$
\int_{\mathbb{R}^{d}} \varphi J d \mathscr{L}^{d}=\int_{\mathbb{R}^{d-k}}\left[\int_{E_{h}} \varphi d \mathscr{H}^{k}\right] d \mathscr{L}^{d-k}(h),
$$

where $J=\left[\operatorname{det}\left(\nabla f \cdot{ }^{t} \nabla f\right)\right]^{1 / 2}$ is the Jacobian of $f$ and $E_{h}=\left\{x \in \mathbb{R}^{d}: f(x)=h\right\}$, for $h \in \mathbb{R}^{d-k}$, are the level sets of the function $f$.

\section{Previous literature and main points of the proof}

We first indicate the essential literature on the two-dimensional transport equation. Previous results by Bouchut and Desvillettes [10], Hauray [24] and Colombini and Lerner $[12,13]$ show that uniqueness holds for the transport equation relative to an autonomous bounded divergence-free vector field, under the following additional condition on the local direction of the vector field: there exists an open set $\Omega \subset \mathbb{R}^{2}$ such that $\mathscr{H}^{1}\left(\mathbb{R}^{2} \backslash \Omega\right)=0$ and for every $x \in \Omega$ the following holds:

$$
\begin{aligned}
& \text { there exist } \xi \in \mathbb{S}^{1}, \alpha>0 \text { and } \varepsilon>0 \text { such that } \\
& \text { for } \mathscr{L}^{2} \text {-a.e. } y \in B_{\varepsilon}(x) \text {, we have } b(y) \cdot \xi \geq \alpha .
\end{aligned}
$$

The validity of this condition permits a local change of variable, which straightens the level sets of the Hamiltonian, thus reducing the equation to a one-dimensional problem (the second spatial variable appears as a parameter in the equation after the change of variable). Some extensions to the non-divergence-free case are due to Colombini and Rauch [14] and to Colombini, Rauch and the third author [11].

However, the meaning of condition (2.1) is not completely clear: while in the stationary problem it just expresses the fact that the surface on which we consider the initial data is noncharacteristic, in the time-space problem it is a kind of local regularity of the direction of $b$. In particular, condition (2.1) prevents the existence of "too many" zeros of the vector field.

The strategy followed in [2] is a bit different: we do not perform a local change of variable according to the Hamiltonian, but we rather split the equation on the level sets of the Hamiltonian, using the coarea formula. Then we would like to look at the equation level set by level set. It turns out that, where $\nabla H \neq 0$, the level sets are in fact nice rectifiable curves, and this will allow to consider the $\mathrm{PDE}$ in the parametrization. The interesting point is that, in order to separate the evolution in $\{\nabla H=0\}$ from the evolution in $\{\nabla H \neq 0\}$, we need a condition which is reminiscent of (2.1), in the sense that it regards again the "amount of critical points of $H$ ". This is precisely the weak Sard property in (4.1). We notice that condition (4.1) is much weaker than (2.1). Surprisingly enough, we are able to show that (4.1) is equivalent to the well-posedness, and we also construct various explicit examples of nonuniqueness when (4.1) is violated. We present in this note an account of the necessity of (4.1) in the basic case of a bounded divergence-free vector field: we remark that various generalizations and extensions are possible (we refer again to [2]). 


\section{Splitting on the level sets of the Hamiltonian}

We are concerned with the Cauchy problem for the transport equation (1.1) and we define the Hamiltonian function $H \in \operatorname{Lip}_{c}\left(\mathbb{R}^{2}\right)$ associated to $b$ as in (1.3). Consider the weak formulation of $(1.1): u(t, x) \in L^{\infty}\left([0, T] \times \mathbb{R}^{2}\right)$ is a weak solution of $(1.1)$ if for every $\varphi(t, x) \in \operatorname{Lip}_{c}\left(\left[0, T\left[\times \mathbb{R}^{2}\right)\right.\right.$ we have

$$
\int_{0}^{T} \int_{\mathbb{R}^{2}} u\left(\partial_{t} \varphi+b \cdot \nabla \varphi\right) d x d t=-\int_{\mathbb{R}^{2}} \bar{u} \varphi(0, \cdot) d x .
$$

Notice that from the coarea formula (1.5) it follows that for every function $\phi \in L^{1}\left(\mathbb{R}^{2}\right)$ we have

$$
\int_{\mathbb{R}^{2} \cap\{\nabla H \neq 0\}} \phi d x=\int_{\mathbb{R}}\left[\int_{E_{h}} \frac{\phi}{|\nabla H|} d \mathscr{H}^{1}\right] d h,
$$

where $E_{h}$ are the level sets of the function $H$. Using (3.2) in (3.1) and recalling that $b=\nabla^{\perp} H$ we obtain

$$
\begin{aligned}
0= & \int_{0}^{T} \int_{\{\nabla H=0\}} u \partial_{t} \varphi d x d t+\int_{\{\nabla H=0\}} \bar{u} \varphi(0, \cdot) d x \\
& +\int_{0}^{T} \int_{\{\nabla H \neq 0\}} u\left(\partial_{t} \varphi+b \cdot \nabla \varphi\right) d x d t+\int_{\{\nabla H \neq 0\}} \bar{u} \varphi(0, \cdot) d x \\
= & \int_{0}^{T} \int_{\{\nabla H=0\}} u \partial_{t} \varphi d x d t+\int_{\{\nabla H=0\}} \bar{u} \varphi(0, \cdot) d x \\
& +\int_{0}^{T} \int_{\mathbb{R}}\left[\int_{E_{h}} \frac{u}{|\nabla H|}\left(\partial_{t} \varphi+b \cdot \nabla \varphi\right) d \mathscr{H}^{1}\right] d h d t \\
& +\int_{\mathbb{R}}\left[\int_{E_{h}} \frac{\bar{u}}{|\nabla H|} \varphi(0, \cdot) d \mathscr{H}^{1}\right] d h .
\end{aligned}
$$

The following lemma will allow the selection of the level sets of the Hamiltonian. We recall that we denote by $H_{\#} \mathscr{L}^{2}$ the push-forward of the Lebesgue measure on $\mathbb{R}^{2}$ via the function $H$, defined according to (1.4).

Lemma 3.1. - If $u(t, x)$ is a weak solution of $(1.1)$ and $\eta(h) \in L^{1}\left(\mathbb{R}, H_{\#} \mathscr{L}^{2}\right)$, then $u(t, x) \eta(H(x))$ is a weak solution of (1.1).

Proof. - Considering the weak formulation (3.1) with test function

$$
\psi(t, x)=\varphi(t, x) \eta(H(x))
$$

we deduce the validity of the lemma for any Lipschitz function $\eta$. The thesis for every $\eta \in L^{1}\left(\mathbb{R}, H_{\#} \mathscr{L}^{2}\right)$ follows from an approximation procedure, since no derivatives of $\eta$ are involved in the weak formulation.

We now introduce some notation that will be used in the rest of this note. We consider the measure $\lambda_{\varphi}$ defined by

$$
\lambda_{\varphi}=H_{\#}\left(\left(\int_{0}^{T} u \partial_{t} \varphi d t+\bar{u} \varphi(0, \cdot)\right) \mathscr{L}^{2}\llcorner\{\nabla H=0\}) .\right.
$$

It is readily checked that $\lambda_{\varphi} \ll H_{\#}\left(\mathscr{L}^{2}\llcorner\{\nabla H=0\})\right.$. We denote by $\lambda_{\varphi}(h)$ the density of $\lambda_{\varphi}$ with respect to $H_{\#}\left(\mathscr{L}^{2}\llcorner\{\nabla H=0\})\right.$, i.e.

$$
\lambda_{\varphi}=\lambda_{\varphi}(h) H_{\#}\left(\mathscr{L}^{2}\llcorner\{\nabla H=0\}) .\right.
$$


Moreover, for every $\eta \in L^{1}\left(\mathbb{R}, H_{\#} \mathscr{L}^{2}\right)$, we have

$$
\lambda_{\eta(H) \varphi}=\eta(h) \lambda_{\varphi}(h) H_{\#}\left(\mathscr{L}^{2}\llcorner\{\nabla H=0\}) .\right.
$$

We now perform the Lebesgue decomposition of $H_{\#}\left(\mathscr{L}^{2}\llcorner\{\nabla H=0\})\right.$ into the absolutely continuous and the singular (with respect to $\mathscr{L}^{1}$ ) parts:

$$
\begin{aligned}
H_{\#}\left(\mathscr{L}^{2}\llcorner\{\nabla H=0\})=\right. & {\left[H_{\#}\left(\mathscr{L}^{2}\llcorner\{\nabla H=0\})\right]^{a}(h) \mathscr{L}^{1}\right.} \\
& +\left[H_{\#}\left(\mathscr{L}^{2}\llcorner\{\nabla H=0\})\right]^{s} .\right.
\end{aligned}
$$

Going back to (3.3), using Lemma 3.1 and the notation introduced we obtain, for every $\eta \in L^{1}\left(\mathbb{R}, H_{\#} \mathscr{L}^{2}\right)$,

$$
\begin{aligned}
& \int_{\mathbb{R}} \eta(h) \lambda_{\varphi}(h) d\left[H_{\#}\left(\mathscr{L}^{2}\llcorner\{\nabla H=0\})\right](h)\right. \\
& +\int_{0}^{T} \int_{\mathbb{R}} \eta(h)\left[\int_{E_{h}} \frac{u}{|\nabla H|}\left(\partial_{t} \varphi+b \cdot \nabla \varphi\right) d \mathscr{H}^{1}\right] d h d t \\
& +\int_{\mathbb{R}} \eta(h)\left[\int_{E_{h}} \frac{\bar{u}}{|\nabla H|} \varphi(0, \cdot) d \mathscr{H}^{1}\right] d h=0 .
\end{aligned}
$$

The arbitrariness of the function $\eta \in L^{1}\left(\mathbb{R}, H_{\#} \mathscr{L}^{2}\right)$ in (3.4) then gives the following:

(i) for $\mathscr{L}^{1}$-a.e. $h \in \mathbb{R}$ we have

$$
\begin{aligned}
& \lambda_{\varphi}(h)\left[H_{\#}\left(\mathscr{L}^{2}\llcorner\{\nabla H=0\})\right]^{a}(h)\right. \\
& +\int_{0}^{T} \int_{E_{h}} \frac{u}{|\nabla H|}\left(\partial_{t} \varphi+b \cdot \nabla \varphi\right) d \mathscr{H}^{1} d t+\int_{E_{h}} \frac{\bar{u}}{|\nabla H|} \varphi(0, \cdot) d \mathscr{H}^{1}=0 ;
\end{aligned}
$$

(ii) for $\left[H_{\#}\left(\mathscr{L}^{2}\llcorner\{\nabla H=0\})\right]^{s}\right.$-a.e. $h \in \mathbb{R}$ we have

$$
\lambda_{\varphi}(h)=0 .
$$

\section{The weak Sard property}

We see from equation (3.5) that the dynamics in $\{\nabla H \neq 0\}$ and in $\{\nabla H=0\}$ could be coupled: this can actually happen, as shown in the examples constructed in [2]. This means that we can have interactions between the areas in which the velocity is zero and the ones in which it is nonzero. In order to separate the two dynamics we need the following weak Sard property of the Hamiltonian. if

Definition 4.1. - We say that $H \in \operatorname{Lip}_{c}\left(\mathbb{R}^{2}\right)$ satisfies the weak Sard property

$$
H_{\#}\left(\mathscr{L}^{2}\llcorner\{\nabla H=0\}) \perp \mathscr{L}^{1} .\right.
$$

Using the notation introduced in the previous section this means that

$$
\left[H_{\#}\left(\mathscr{L}^{2}\llcorner\{\nabla H=0\})\right]^{a}(h)=0 \quad \text { for } \mathscr{L}^{1} \text {-a.e. } h \in \mathbb{R}\right. \text {. }
$$

The connection with the classical Sard theorem (see for instance Theorem 3.4.3 of [23]) is evident: here we are requiring that the "image" (via the push-forward 
through $H$ ) of the Lebesgue measure $\mathscr{L}^{2}$ restricted to the set of the critical points $\{\nabla H=0\}$ is "not seen" by the Lebesgue measure $\mathscr{L}^{1}$ in the codomain.

Assuming the weak Sard property we can separate the two dynamics, hence from equations (3.5) and (3.6) we deduce the following result.

THEOREM 4.2. - Let $b \in L^{\infty}\left(\mathbb{R}^{2} ; \mathbb{R}^{2}\right)$ with compact support and assume that $\operatorname{div} b=0$. Let $H \in \operatorname{Lip}_{c}\left(\mathbb{R}^{2}\right)$ be as in (1.3) and assume that $H$ satisfies the weak Sard property (4.1). Let $u \in L^{\infty}\left([0, T] \times \mathbb{R}^{d}\right)$ be a weak solution of (1.1). Then we have

$$
\int_{0}^{T} \int_{E_{h}} \frac{u}{|\nabla H|}\left(\partial_{t} \varphi+b \cdot \nabla \varphi\right) d \mathscr{H}^{1} d t+\int_{E_{h}} \frac{\bar{u}}{|\nabla H|} \varphi(0, \cdot) d \mathscr{H}^{1}=0
$$

for $\mathscr{L}^{1}$-a.e. $h \in \mathbb{R}$ and

$$
\int_{0}^{T} \int_{\{\nabla H=0\}} u \partial_{t} \varphi d x d t+\int_{\{\nabla H=0\}} \bar{u} \varphi(0, \cdot) d x=0 .
$$

Notice that (4.3) gives $u(t, x)=\bar{u}$ for $\mathscr{L}^{1} \otimes \mathscr{L}^{2}$-a.e. $(t, x) \in[0, T] \times\{\nabla H=0\}$. This means that, thanks to the weak Sard property, the uniqueness for the Cauchy problem (1.1) is equivalent to the uniqueness for the "reduced problems" (4.2) on the level sets, for $\mathscr{L}^{1}$-a.e. $h \in \mathbb{R}$. The issue of the uniqueness on the level sets is discussed in Section 6.

\section{Structure of the level sets}

In this section we give a detailed description of the structure of the level sets

$$
E_{h}=\left\{x \in \mathbb{R}^{2}: H(x)=h\right\} .
$$

We first notice, by the continuity of $H$ and by the assumption of compactness of the support, that for every $h \neq 0$ the set $E_{h}$ is compact. Moreover, an application of the coarea formula in the form given in (3.2) gives

$$
\int_{E_{h}} \frac{1}{|\nabla H|} d \mathscr{H}^{1}<+\infty \quad \text { for } \mathscr{L}^{1} \text {-a.e. } h \in \mathbb{R} \text {. }
$$

In particular, since $|\nabla H| \leq\|b\|_{\infty}$, this yields

$$
\mathscr{H}^{1}\left(E_{h}\right)<+\infty \text { for } \mathscr{L}^{1} \text {-a.e. } h \in \mathbb{R} \text {. }
$$

For every $h \in \mathbb{R}$, we denote by $\mathscr{C}_{h}$ the family of all the connected components $C$ of $E_{h}$ such that $\mathscr{H}^{1}(C)>0$ (in fact, these are just the connected components which contain more than one point).

We collect together in the following theorem all the results relative to the classification of the level sets. For the proof we refer to [2]. See the end of the first section for the notion of rectifiable set. hold.

TheOREm 5.1. - Let $H \in \operatorname{Lip}_{c}\left(\mathbb{R}^{2}\right)$. For $\mathscr{L}^{1}$-a.e. $h \in \mathbb{R}$ the following statements

(i) $E_{h}$ is $\mathscr{H}^{1}$-rectifiable and $\mathscr{H}^{1}\left(E_{h}\right)<+\infty$; the map $H$ is differentiable in $x$ and $\nabla H \neq 0$ at $\mathscr{H}^{1}$-a.e. $x \in E_{h}$; the function $1 /|\nabla H|$ belongs to $L^{1}\left(E_{h}, \mathscr{H}^{1}\right)$.

(ii) The family $\mathscr{C}_{h}$ is countable and $\mathscr{H}^{1}\left(E_{h} \backslash \cup_{C \in \mathscr{C}_{h}} C\right)=0$. 
(iii) Every $C \in \mathscr{C}_{h}$ is a closed simple curve. More precisely, it is possible to find a Lipschitz injective parametrization $\gamma:[\alpha, \beta]^{*} \rightarrow C$ such that

$$
\dot{\gamma}(s)=\nabla^{\perp}(\gamma(s)) \quad \text { for } \mathscr{L}^{1} \text {-a.e. } s \in[\alpha, \beta]^{*},
$$

where we denote by $[\alpha, \beta]^{*}$ the quotient space consisting of the interval $[\alpha, \beta]$ with identified endpoints, endowed with the distance

$$
\operatorname{dist}_{[\alpha, \beta]^{*}}(x, y)=\min \{|x-y|,(\beta-\alpha)-|x-y|\} .
$$

We will also need the following topological lemma.

Lemma 5.2. - Let $h \in \mathbb{R}$ such that the conclusions of Theorem 5.1 hold. Then, for every $C \in \mathscr{C}_{h}$, there exists a decreasing sequence $\left\{U_{n}\right\}$ of bounded open sets in $\mathbb{R}^{2}$ such that $\partial U_{n} \cap E_{h}=\emptyset$ for every $n$ and $E_{h} \cap\left(\cap_{n} U_{n}\right)=C$.

\section{Uniqueness on the level sets and conclusion of the proof}

We first show, using Lemma 5.2, that equation (4.2) can be separated into a family of equations on the connected components of each level set.

Proposition 6.1. - Let $b \in L^{\infty}\left(\mathbb{R}^{2} ; \mathbb{R}^{2}\right)$ with compact support and assume that $\operatorname{div} b=0$. Let $H \in \operatorname{Lip}_{c}\left(\mathbb{R}^{2}\right)$ be as in (1.3) and assume that $H$ satisfies the weak Sard property (4.1). Let $u \in L^{\infty}\left([0, T] \times \mathbb{R}^{d}\right)$ be a weak solution of (1.1). Then, for every $C \in \mathscr{C}_{h}$, for $\mathscr{L}^{1}$-a.e. $h \in \mathbb{R}$, we have

$$
\int_{0}^{T} \int_{C} \frac{u}{|\nabla H|}\left(\partial_{t} \varphi+b \cdot \nabla \varphi\right) d \mathscr{H}^{1} d t+\int_{C} \frac{\bar{u}}{|\nabla H|} \varphi(0, \cdot) d \mathscr{H}^{1}=0 .
$$

Proof. - We fix $h \in \mathbb{R}$ such that the conclusions of Theorems 4.2 and 5.1 hold. We choose a sequence $\left\{U_{n}\right\}$ as in Lemma 5.2. Since $\partial U_{n}$ and $E_{h}$ are compact sets we have

$$
\operatorname{dist}\left(\partial U_{n}, E_{h}\right)=\varepsilon_{n}>0 .
$$

Thus we fix a standard convolution kernel $\rho$ with spt $\rho \subset B_{1}(0)$ and for every $n$ we set

$$
\gamma_{n}(x)=\mathbf{1}_{U_{n}} * \rho_{\varepsilon_{n} / 4}(x),
$$

where we denote with $\mathbf{1}_{U_{n}}$ the characteristic function of the set $U_{n}$. We rewrite equation (4.2) with the test function $\varphi(t, x) \gamma_{n}(x)$. We have

$$
\begin{aligned}
0 & =\int_{0}^{T} \int_{E_{h}} \frac{u}{|\nabla H|}\left(\partial_{t} \varphi \gamma_{n}+b \cdot \nabla\left(\varphi \gamma_{n}\right)\right) d \mathscr{H}^{1} d t+\int_{E_{h}} \frac{\bar{u}}{|\nabla H|} \varphi(0, \cdot) \gamma_{n} d \mathscr{H}^{1} \\
& =\int_{0}^{T} \int_{E_{h} \cap U_{n}} \frac{u}{|\nabla H|}\left(\partial_{t} \varphi+b \cdot \nabla \varphi\right) d \mathscr{H}^{1} d t+\int_{E_{h} \cap U_{n}} \frac{\bar{u}}{|\nabla H|} \varphi(0, \cdot) d \mathscr{H}^{1} .
\end{aligned}
$$

We now let $n \rightarrow \infty$ in the above equality. Recalling (5.1) and applying the Lebesgue dominated convergence theorem we eventually obtain (6.1).

We are now in the position to formulate and prove our main result.

THEOREM 6.2. - Let $b \in L^{\infty}\left(\mathbb{R}^{2} ; \mathbb{R}^{2}\right)$ with compact support and assume that $\operatorname{div} b=0$. Let $H \in \operatorname{Lip}_{c}\left(\mathbb{R}^{2}\right)$ be as in (1.3) and assume that $H$ satisfies the weak Sard property (4.1). Then, for every initial data $\bar{u} \in L^{\infty}\left(\mathbb{R}^{2}\right)$, the Cauchy problem (1.1) has a unique solution $u \in L^{\infty}\left([0, T] \times \mathbb{R}^{2}\right)$. 
We remark that from this theorem it also follows a well-posedness result for the ordinary differential equation (1.2). In this low regularity context, existence and uniqueness for the ODE are proved (via some well-established abstract arguments, starting from the uniqueness result for the PDE, see for instance [7]) in the context of the so-called regular Lagrangian flows, i.e. roughly speaking ODE flows that "compress the trajectories in a uniformly controlled way".

Proof of Theorem 6.2. - The existence is obtained by a standard regularization technique, see for instance [7]. To show the uniqueness, by linearity it suffices to show that the only solution $u \in L^{\infty}\left([0, T] \times \mathbb{R}^{2}\right)$ with initial data $\bar{u} \equiv 0$ is $u \equiv 0$. Recalling the discussion at the end of Section 4 and the result of Proposition 6.1, it is enough to show that, for every $C \in \mathscr{C}_{h}$, for $\mathscr{L}^{1}$-a.e. $h \in \mathbb{R}$, the validity of

$$
\int_{0}^{T} \int_{C} \frac{u}{|\nabla H|}\left(\partial_{t} \varphi+b \cdot \nabla \varphi\right) d \mathscr{H}^{1} d t=0
$$

for every $\varphi(t, x) \in \operatorname{Lip}_{c}\left(\left[0, T\left[\times \mathbb{R}^{2}\right)\right.\right.$ implies

$$
u(t, x)=0 \quad \text { for } \mathscr{L}^{1} \otimes \mathscr{H}^{1} \text {-a.e. }(t, x) \in[0, T] \times C .
$$

We proceed in several steps.

Step 1. Parametrization of $C$. - We fix $h \in \mathbb{R}$ such that the conclusions of Theorem 5.1 and of Proposition 6.1 hold. Using the result in Theorem 5.1(iii) we know that every $C \in \mathscr{C}_{h}$ is a closed simple curve and we choose a Lipschitz injective parametrization $\gamma:[\alpha, \beta]^{*} \rightarrow C$ which satisfies (5.2). Hence from (6.1) (with $\bar{u} \equiv 0$ ) we get

$$
\int_{0}^{T} \int_{\alpha}^{\beta} u(t, \gamma(s))\left(\left(\partial_{t} \varphi\right)(t, \gamma(s))+b(\gamma(s)) \cdot(\nabla \varphi)(t, \gamma(s))\right) d s d t=0
$$

for every $\varphi \in \operatorname{Lip}_{c}\left(\left[0, T\left[\times \mathbb{R}^{2}\right)\right.\right.$. We set

$$
\tilde{\varphi}(t, s)=\varphi(t, \gamma(s)) .
$$

Differentiating both sides of (6.4) with respect to $s$ we get

$$
\partial_{s} \tilde{\varphi}(t, s)=(\nabla \varphi)(t, \gamma(s)) \cdot \dot{\gamma}(s)=(\nabla \varphi)(t, \gamma(s)) \cdot\left(\nabla^{\perp} H\right)(\gamma(s)),
$$

and this implies

$$
b(\gamma(s)) \cdot(\nabla \varphi)(t, \gamma(s))=\left(\nabla^{\perp} H\right)(\gamma(s)) \cdot(\nabla \varphi)(t, \gamma(s))=\partial_{s} \tilde{\varphi}(t, s) .
$$

Setting $\tilde{u}(t, s)=u(t, \gamma(s))$ and inserting (6.5) in (6.3) we obtain

$$
\int_{0}^{T} \int_{\alpha}^{\beta} \tilde{u}\left(\partial_{t} \tilde{\varphi}+\partial_{s} \tilde{\varphi}\right) d s d t=0
$$

for every $\tilde{\varphi}:[0, T] \times[\alpha, \beta]^{*} \rightarrow \mathbb{R}$ of the form $\tilde{\varphi}(t, s)=\varphi(t, \gamma(s))$ for some $\varphi \in$ $\operatorname{Lip}_{c}\left(\left[0, T\left[\times \mathbb{R}^{2}\right)\right.\right.$.

Step 2. Test functions in $[0, T] \times[\alpha, \beta]^{*}$. - We notice that, up to now, we cannot see (6.6) as a distributional equation on $[0, T] \times[\alpha, \beta]^{*}$ : indeed, we are allowed to use as test functions only the particular $\tilde{\varphi}$ 's of the form above. However, the following lemma from [2] holds. 
LEMMA 6.3. - Every $\psi \in \operatorname{Lip}_{c}\left(\left[0, T\left[\times[\alpha, \beta]^{*}\right)\right.\right.$ can be approximated uniformly with a sequence of functions $\left\{\tilde{\varphi}_{n}\right\}$ of the form above and such that $\operatorname{Lip}\left(\tilde{\varphi}_{n}\right)$ is equi-bounded.

This means that we can write (6.6) with $\tilde{\varphi}=\tilde{\varphi}_{n}$ for every $n$ and passing to the limit we get

$$
\int_{0}^{T} \int_{\alpha}^{\beta} \tilde{u}\left(\partial_{t} \psi+\partial_{s} \psi\right) d s d t=0
$$

for any $\psi \in \operatorname{Lip}_{c}\left(\left[0, T\left[\times,[\alpha, \beta]^{*}\right)\right.\right.$. This is now a distributional equation on $[0, T] \times$ $[\alpha, \beta]^{*}$.

Step 3. Uniqueness on $C$. - Now it suffices to notice that (6.7) is the distributional form of the Cauchy problem

$$
\left\{\begin{array}{l}
\partial_{t} \tilde{u}+\partial_{s} \tilde{u}=0 \\
\tilde{u}(0, \cdot)=0
\end{array}\right.
$$

By the smooth theory for the transport equation (see [7]) we know that the only solution to this problem is $\tilde{u} \equiv 0$. From the definition of $\tilde{u}$ we see that this precisely implies (6.2), thus we have shown the desired thesis.

We close this note by presenting two particular cases in which the weak Sard property (4.1) is satisfied by the function $H \in \operatorname{Lip}_{c}\left(\mathbb{R}^{2}\right)$ associated to $b$ as in (1.3) and thus the uniqueness result of Theorem 6.2 holds. See [2] for the proof.

Corollary 6.4. - Let $b \in L^{\infty}\left(\mathbb{R}^{2} ; \mathbb{R}^{2}\right)$ with compact support and assume that $\operatorname{div} b=0$ and that $b$ is approximately differentiable $\mathscr{L}^{2}$-a.e. in $\mathbb{R}^{2}$. Then, for every initial data $\bar{u} \in L^{\infty}\left(\mathbb{R}^{2}\right)$, the Cauchy problem (1.1) has a unique solution $u \in L^{\infty}\left([0, T] \times \mathbb{R}^{2}\right)$.

We observe that the approximate differentiability assumption on $b$ in Corollary 6.4 is of "qualitative" type, in contrast with the usual "quantitative" weak regularity assumptions, for instance Sobolev or $B V$. In the second corollary we deal with the case in which we assume a control on the distributional curl of the vector field.

Corollary 6.5. - Let $b \in L^{\infty}\left(\mathbb{R}^{2} ; \mathbb{R}^{2}\right)$ with compact support and assume that $\operatorname{div} b=0$ and that the distributional curl of $b$ is a measure. Then, for every initial data $\bar{u} \in L^{\infty}\left(\mathbb{R}^{2}\right)$, the Cauchy problem (1.1) has a unique solution $u \in L^{\infty}([0, T] \times$ $\left.\mathbb{R}^{2}\right)$.

\section{References}

1. M. Aizenman: On vector fields as generators of flows: a counterexample to Nelson's conjecture. Ann. Math. 107 (1978), 287-296.

2. G. Alberti, S. Bianchini, G. Crippa: Work in preparation.

3. L. Ambrosio: Transport equation and Cauchy problem for $B V$ vector fields. Invent. Math. 158 (2004), 227-260.

4. L. Ambrosio: Transport equation and Cauchy problem for non-smooth vector fields and applications. Calculus of variations and nonlinear partial differential equations (Lectures given at the C.I.M.E. Summer School held in Cetraro, June 27-July 2, 2005. Edited by B. Dacorogna and P. Marcellini), pp. 1-41. Lecture Notes in Math., 1927. Springer, Berlin 2008.

5. L. Ambrosio, F. Bouchut, C. De Lellis: Well-posedness for a class of hyperbolic systems of conservation laws in several space dimensions. Comm. Partial Differential Equations 29 (2004), 1635-1651.

6. L. Ambrosio, C. De Lellis: Existence of solutions for a class of hyperbolic systems of conservation laws in several space dimensions. Int. Math. Res. Not. no. 41 (2003), 2205-2220. 
7. L. Ambrosio, G. Crippa: Existence, uniqueness, stability and differentiability properties of the flow associated to weakly differentiable vector fields. Transport equations and multi-D hyperbolic conservation laws (Lecture notes from the Winter School held in Bologna, January 2005. Edited by F. Ancona et al.), pp. 3-57. Lect. Notes Unione Mat. Ital., 5. Springer, Berlin 2008.

8. L. Ambrosio, N. Fusco, D. Pallara: Functions of bounded variation and free discontinuity problems. Oxford Mathematical Monographs. Oxford University Press, Oxford 2000.

9. A. Bressan: An ill posed Cauchy problem for a hyperbolic system in two space dimensions. Rend. Sem. Mat. Univ. Padova 110 (2003), 103-117.

10. F. Bouchut, L. Desvillettes: On two-dimensional Hamiltonian transport equations with continuous coefficients. Differential Integral Equations 14 (2001), 1015-1024.

11. F. Colombini, G. Crippa, J. Rauch: A note on two-dimensional transport with bounded divergence. Comm. Partial Differential Equations 31 (2006), 1109-1115.

12. F. Colombini, N. Lerner: Sur les champs de vecteurs peu réguliers. Séminaire: Équations aux Dérivées Partielles 2000/2001, Exp. No. XIV. École Polytech., Palaiseau 2001.

13. F. Colombini, N. Lerner: Uniqueness of $L^{\infty}$ solutions for a class of conormal $B V$ vector fields. Contemp. Math. 368 (2005), 133-156.

14. F. Colombini, J. Rauch: Uniqueness in the Cauchy Problem for Transport in $\mathbb{R}^{2}$ and $\mathbb{R}^{1+2}$. J. Differential Equations 211 (2005), 162-167.

15. G. Crippa, C. De Lellis: Estimates and regularity results for the DiPerna-Lions flow. J. Reine Angew. Math. 616 (2008), 15-46.

16. C. Dafermos: Hyperbolic conservation laws in continuum physics. Second edition. Grundlehren der Mathematischen Wissenschaften, 325. Springer-Verlag, Berlin, 2005.

17. C. De Lellis: Notes on hyperbolic systems of conservation laws and transport equations. Handbook of Differential Equations: Evolutionary Equations, vol. III (Edited by C. M. Dafermos and E. Feireisl), pp. 277-382. Elsevier/North-Holland, Amsterdam 2007.

18. C. De Lellis: Ordinary differential equations with rough coefficients and the renormalization theorem of Ambrosio (d'après Ambrosio, DiPerna, Lions). Séminaire Bourbaki 2006/2007. Astérisque 317 (2008), Exp. No. 972, viii, 175-203.

19. N. Depauw: Non unicité des solutions bornées pour un champ de vecteurs $B V$ en dehors d'un hyperplan. C.R. Math. Sci. Acad. Paris 337 (2003), 249-252.

20. R.J. DiPerna, P.-L. Lions: Ordinary differential equations, transport theory and Sobolev spaces. Invent. Math. 98 (1989), 511-547.

21. R. Engelking: General Topology. Revised and completed edition. Sigma series in pure mathematics, 6. Heldermann Verlag, Berlin 1989.

22. L.C. Evans, R.F. Gariepy: Measure theory and fine properties of functions. Studies in Advanced Mathematics. CRC Press, Boca Raton 1992.

23. H. Federer: Geometric measure theory. Die Grundlehren der mathematischen Wissenschaften, 153. Springer-Verla, New York 1969.

24. M. Hauray: On two-dimensional Hamiltonian transport equations with $L_{\text {loc }}^{p}$ coefficients. Ann. Inst. H. Poincaré Anal. Non Linéaire 20 (2003), 625-644.

25. B.L. Keyfitz, H.C. Kranzer: A system of nonstrictly hyperbolic conservation laws arising in elasticity theory. Arch. Rational Mech. Anal. 72 (1979/80), 219-241.

26. D. Serre: Systèmes de lois de conservation. I, II. Diderot Editeur, Paris 1996.

27. W.P. Ziemer: Weakly differentiable functions. Sobolev spaces and functions of bounded variation. Graduate Texts in Mathematics, 120. Springer-Verlag, New York 1989.

G.A.

Dipartimento di Matematica, Università degli Studi di Pisa, largo Pontecorvo 5, 56127 Pisa, Italy.

E-mail: galberti1@dm.unipi.it

S.B.

SISSA-ISAS, via Beirut 4, 34014 Trieste, Italy.

E-mail: bianchin@sissa.it

G.C.

Dipartimento di Matematica, Università degli Studi di Parma, viale G.P. Usberti 53/A (Campus), 43100

Parma, Italy.

E-mail: gianluca.crippa@unipr.it 